\title{
Evaluación cualitativa de indicadores de sostenibilidad socioambiental para su selección y aplicación en ciudades costarricenses
}

\author{
Qualitative evaluation of socio-environmental sustainability \\ indicators for their selection and application in Costa Rican cities
}

\author{
Marilyn Romero-Vargas ${ }^{1}$ \\ Tania Bermúdez-Rojas ${ }^{2}$ \\ Universidad Nacional, Costa Rica \\ Maritza Duque-Gutiérrez ${ }^{3}$ \\ Universidad del Norte, Colombia
}

\begin{abstract}
Resumen
Desde el modelo de gestión del desarrollo sostenible, las ciudades deben procurar unas relaciones de equilibrio entre los recursos naturales y el desarrollo económico y social, de tal forma que las condiciones ambientales dentro y fuera de estos espacios urbanos no alcancen patrones y condiciones no deseadas. Este estudio surge de la necesidad de contar con un sistema de seguimiento de la sostenibilidad socioambiental de las ciudades por medio de criterios e indicadores. Con la participación de expertos nacionales se generó una lista 327 indicadores, los cuales fueron reagrupados según sus características en 82 indicadores distribuidos en 9 criterios: agua, energía, fauna, áreas verdes urbanas, suelo, gestión ambiental, manejo de residuos sólidos y líquidos, ordenamiento del territorio y sociedad \& gobernanza. Por medio de revisión bibliográfica, solo 43 indicadores contaron con información completa y operativa para ser evaluados. Mediante taller de expertos nacionales se evalúo la factibilidad teórica de ser aplicados. El resultado final es un compendio de 19 indicadores socioambientales, que cumplen con al menos el $80 \%$ de pertinencia, relevancia, robustez y disponibilidad de datos. Sin embargo, los expertos mencionaron que el costo, la escala

1 Doctora en Geografía. Académica de la Escuela de Ciencias Geográficas de la Universidad Nacional Costa Rica correo electrónico mari.romerov@gmail.com

2 Master of Sciencie, Bióloga. Académica de la Escuela Ciencias Biológicas de la Universidad Nacional Costa Rica correo electrónico tania.bermudez.rojas@una.cr

3 Doctora en Tecnología Ambiental y Gestión del Agua. Académica de la Universidad del Norte de Colombia correo electrónico maritzaduque@uninorte.edu.co
\end{abstract}


Marilyn Romero-Vargas - Tania Bermúdez-Rojas - Maritza Duque-Gutiérrez

Evaluación cualitativa de indicadores de sostenibilidad socioambiental para su selección y aplicación en ciudades costarricenses

y la calidad son variables que deben ser utilizadas para seleccionar, adaptar o construir indicadores que permitan el seguimiento de la sostenibilidad socioambiental para ciudades de Costa Rica.

Palabras clave: indicadores socioambientales, sostenibilidad ecológica, ciudades costarricenses.

\begin{abstract}
From the sustainable development management model, cities must seek balance relationships between natural resources and economic and social development in such a way that the environmental conditions inside and outside these urban spaces do not reach undesirable patterns and conditions. This study arises from the need to possess a system for monitoring the socio-environmental sustainability of cities through criteria and indicators. With the participation of national experts, a preliminary list of 327 indicators was generated which were then grouped based on their characteristics into 82 indicators distributed along 9 distinctive criteria, namely: water, energy, fauna, urban green areas, soil, environmental management, solid and liquid waste management, land use planning and society \& governance. By means of bibliographic review, only 43 indicators were found to hold complete and operative information to be evaluated. Through a participative workshop involving the national experts, the theoretical feasibility of applying the selected indicators was evaluated. The final result is a compendium of 19 socio-environmental indicators, which comply with at least $80 \%$ pertinence, relevance, robustness and data availability. However, the experts point out that cost, scale and quality are all variables that must be used in selecting, adapting or creating indicators that allow for the monitoring of socio-environmental sustainability in Costa Rican cities.
\end{abstract}

Keywords: Socio-environmental indicators; Ecological sustainability; Costa Rican cities.

\title{
Introducción
}

Las ciudades, hábitat de la especie humana (homo urbanis; Gleeson 2013:1841), son desde el punto de vista ecológico, ecosistemas heterótrofos (Odum, 1971), es decir, su organización depende del aporte continuo de agua, materiales y energía que obtienen de ecosistemas adyacentes, sean estos locales, regionales o globales. Esta dependencia del sistema se ha visto agravada en la época del Antropoceno (Crutzen, 2010), cuyas ciudades se caracterizan por acelerados procesos de urbanización, rápido crecimiento poblacional, estilos de vida consumista y dificultades de gestión de los residuos.

La urbanización como proceso destruye y fragmenta ecosistemas naturales con la consiguiente pérdida de biodiversidad, modificación de los flujos de agua, energía y nutrientes. Consume dos terceras partes de la energía mundial, secuestra considerables proporciones de servicios de los ecosistemas, introduce especies invasoras, expulsa grandes cantidades de residuos y emisiones a la atmósfera (Montes y Duque, 2015:2). 
Los espacios urbanos en general y las ciudades costarricenses en particular están distribuidos en todo el territorio nacional, sin embargo, por tamaño, densidad urbanística, cantidad de población y densidad poblacional, la mayor concentración urbana se ubica en el interior del país, conformando la región Gran Área Metropolitana (GAM) localizada en el Valle Central; una región de aproximadamente $177979 \mathrm{Km}^{2}$ (3,78 \% del territorio nacional) pero con más de la mitad $(52,7 \%)$ de la población total del país, para una densidad poblacional de 70 hab/Ha (Consejo Nacional de Planificación Urbana, 2013, p I-43; Decreto Ejecutivo N³8145 2014). La GAM, con sus cuatro áreas metropolitanas: San José, Alajuela, Heredia y Cartago, presentan un patrón de conurbación urbana a partir de la expansión horizontal de sus cabeceras de cantón (distritos centrales), un modelo de ciudad polinuclear desconcentrado conceptualizado en el Plan GAM de 1982, que con el nuevo Plan GAM 2013-2030 (Decreto Ejecutivo $\mathrm{N}^{\circ} 38145$ 2014) busca reorientar su desarrollo urbano hacia un modelo de centralidades densas e integrales, es decir, un modelo de ciudad regional (Consejo Nacional de Planificación Urbana, 2013 p 20),

Además de la GAM, existen ciudades de menor tamaño en otras regiones del país, localizadas, tanto en el interior como en zonas costeras, entre las que destacan las ciudades costeras Puntarenas, Limón y Jacó, así como Liberia, Ciudad Quesada, San Isidro de El General y Guápiles como ciudades del interior.

De acuerdo con el Estado de la Nación (2015), entre 1982 y 2013 el área construida de la GAM casi se duplicó, debido, principalmente, a la dinámica del mercado inmobiliario. El Estado de la Nación señala, en su capítulo "Armonía con la Naturaleza", las implicaciones de la falta de ordenamiento territorial en la GAM y reconocen que el modelo de ciudad costarricense carece de visión regional y ha provocado severos efectos ambientales, económicos y sociales debido a patrones de crecimiento desordenado y débiles o nulas políticas de gestión del suelo. Lo más grave, es, según el Estado de la Nación (Programa Estado de la Nación, 2017), que las ciudades intermedias como San Isidro del General, Guápiles y Ciudad Quesada, están reproduciendo los errores de la GAM, inclusive un crecimiento más acelerado y disperso, problemas de red de saneamiento, gestión de residuos, congestionamiento vehicular, contaminación atmosférica 
Marilyn Romero-Vargas - Tania Bermúdez-Rojas - Maritza Duque-Gutiérrez Evaluación cualitativa de indicadores de sostenibilidad socioambiental para su selección y aplicación en ciudades costarricenses

asociada a los hidrocarburos, cambios en el uso del suelo, escasez y contaminación de agua, ocupación de áreas vulnerables, etc.

Según este informe del Estado de la Nación (2017), con la excepción de las áreas de conservación, Costa Rica no tiene una prioridad ambiental en la forma de planificar la ocupación del suelo, ni el aprovechamiento de los recursos naturales, por lo que "en el mediano y largo plazo prevalecen tendencias insostenibles" y aunque también reportan fortalezas históricas y avances específicos como la recuperación de la cobertura forestal, electricidad limpia, acceso al agua potable, herramientas legales e instrumentos pioneros para la conservación, el mensaje del Programa es enfático al señalar que: "estos logros no alcanzan para cambiar el rumbo de los patrones de uso del territorio y su falta de ordenamiento, el aprovechamiento de los recursos naturales clave para el desarrollo (como energía y el agua) y la gestión política." (Programa Estado de la Nación, 2017: 55)

Las ciudades pueden ser conceptuadas desde dos enfoques distintos: desde un ecosistema urbano o desde un metabolismo urbano. Aunque ambos son conceptualmente distintos, ellos comparten atributos que son esencialmente importantes en el manejo y gestión de las ciudades, lo que permite la combinación de estos (Bai, 2016), orientando la ciudad hacia la sostenibilidad. Basados en la ciudad como un sistema metabólico, es importante que este trascienda de un sistema lineal (de la cuna a la muerte) a uno cíclico, lo cual significa que los insumos que entran al sistema, por ejemplo, agua y energía, deben contar con acciones estratégicas de manejo y gestión que disminuyan las salidas de residuos, vertidos y emisiones (Figura 1). Sin embargo, la ciudad nunca será sostenible en sentido ecológico, debido a su dependencia de otros sistemas. Por tanto, desde el modelo de ciudad sostenible, su sostenibilidad es un horizonte definido por los objetivos normativos sociales que se persiguen.

Lo anterior implica que la ciudad debe ser pensada como un sistema complejo, impredecible en su trayectoria y de efectos multiescalares (Montes y Duque 2015), el cual requiere de instrumentos de evaluación que permitan medir la calidad del sistema, para la toma de decisiones y así establecer acciones estratégicas de manejo y gestión. Estos instrumentos pueden ser indicadores enfocados desde el concepto metabólico cíclico, acompañados con algunos aspectos de ecosistema urbano. 
Los indicadores son un instrumento de toma de decisiones, ya que estos presentan características observables y muchas veces asociadas a un dato estadístico, lo cual permite el análisis y seguimiento de las acciones (Niemi y McDonald 2004). Además, los indicadores generan información numérica, precisa y contextualizada que puede aplicarse en el ámbito social, económico, ambiental y urbanístico, según las necesidades y objetivos planteados para su uso (Rueda, 1999; Quiroga, 2009; Rueda, S., De Cáceres, R., Cuchí, A., \& Brau, L., 2012).

Por otro lado, la selección de los indicadores es de suma importancia y cuidado para medir realmente lo que se busca. En este sentido, la comunidad internacional señala que un indicador es considerado funcional si cumple con un conjunto de características, tales como: ser medible, de bajo costo, fácil de comprender, ser exacto, tener capacidad de síntesis, entre otras (Rueda 1999, Feria 2003, Niemi y McDonald 2004, Hernández 2009, Morales- Cerdas 2017).

Sumado a lo anterior, los indicadores se basan en una dimensión de interés dentro de un marco de acción, con un conjunto de criterios que se agrupan según sus similitudes (Escobar, 2006). El presente estudio está enfocado hacia la sostenibilidad de las ciudades costarricenses desde una dimensión socioambiental, seleccionando cualitativamente, mediante criterio de expertos indicadores que permitan evaluar la calidad de la ciudad desde un sistema metabólico cíclico, pero también incorporando atributos del ecosistema urbano (Bai 2016) (Figura 1). Definimos para este estudio criterio e indicador socioambiental, como aquel que permite medir la interrelación sociedad, ambiente, cultura y naturaleza producto del uso, manejo y gestión de los recursos naturales. 
Figura 1. Diagrama conceptual para la aplicación de indicadores de evaluación socioambiental del manejo y la gestión de la ciudad, combinando los enfoques de metabolismo cíclico y ecosistema urbano

(Basado en Bai 2016).

\section{INDICADORES SOCIOAMBIENTALES

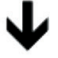

ENTRADAS:

AGUA

ENERGIA

ALIMENTOS

MATERIALES

PERSONAS, ETC

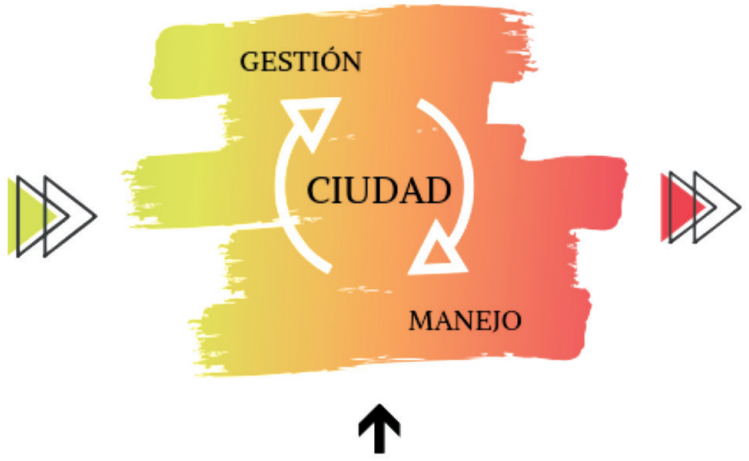

\section{SALIDAS:}

\section{EMISIONES}

RESIDUOS

VERTIDOS

CONOCIMIENTO SERVICIOS, ETC.

INDICADORES SOCIOAMBIENTALES

Fuente: Elaboración propia basado en Bai 2016.

\section{Metodología}

La selección de indicadores socioambientales se realizó por medio de la metodología de criterio de experto, este es un método válido, ya que considera la experiencia de los técnicos y profesionales que diariamente gestionan y manejan el territorio que comprenden las ciudades. Este método, tiene la capacidad de recopilar información sobre un tema particular, a través de expertos que están físicamente alejados, además, genera información válida con respuestas abiertas y bien estructuradas (Luna et al, 2005, Blasco et al, 2010, Munier, 2011).

La consulta a los expertos se realizó por medio de una encuesta vía web, enviada a 87 investigadores nacionales de diferentes disciplinas. La selección de estos expertos se realizó por medio de la metodología de "bola de nieve", que se basa en muestreos en cadena no probabilísticas, en donde los investigadores, luego de contactar al primer sujeto le pide ayuda a él para identificar otras personas que tengan el rasgo o la información afín al tema a investigar (Corral et al, 2015). 
En busca de la multidisciplinariedad, los expertos consultados laboran en instituciones públicas y privadas en temas relacionados con planificación urbana, gestión ambiental o ecología urbana. La encuesta fue completada por 40 expertos lo que representa un $45 \%$ de respuesta.

La información obtenida en la encuesta fue recopilada, sistematizada y ordenada. Asimismo, estos indicadores fueron agrupados en criterios, basados en las similitudes de aspectos que evalúa cada uno, tomando como referencia la literatura disponible, así como bases de datos digitales (Academic Search Complete, Annual Reviews, Biological Abstracts, Current Contents, Environment Complete, JSTOR, Science, Scopus, Springer, Web of Science), permitiendo con esto obtener la operacionalización de los indicadores. Cada indicador se detalló en fichas de sistematización la cual incluyó nombre, descripción, método de medición, escala y las referencias pertinentes de cada indicador (Quiroga 2009).

Seguidamente, se realizaron dos talleres con 15 y 10 expertos, respectivamente, que participaron en la encuesta. En estos talleres se presentó la lista preliminar de indicadores, agrupados en criterios y con información sistematizada y completa para su análisis. Asimismo, los participantes en estos talleres fueron divididos en dos grupos de trabajo, con la finalidad de calificar cualitativamente la factibilidad de cada indicador de ser aplicado en ciudades costarricenses. Esto se efectuó por medio de un instrumento de evaluación teórica por parte de los especialistas, que incluyó nueve variables (Cuadro 1). (Morales - Cerdas, 2017 construido a partir de: Rueda, 1999, Niemi y McDonald, 2004, Feria 2003, Hernández, 2009).

Las variables consideradas presentan una escala de uno a cinco, siendo: 1 muy baja, 2 baja, 3 regular, 4 alta y 5 muy alta. La puntuación máxima por alcanzar por cada indicador fue de 45 puntos (Morales-Cerdas, 2017). A partir de este puntaje se determinó el valor de factibilidad para cada indicador, seleccionando aquellos con un valor mayor o igual a 36 puntos como factibles para ser aplicados en ciudades costarricenses.

Por último, se sistematizó aquellos indicadores con un puntaje mayor o igual a 36 puntos, equivalente al $80 \%$, y se incluyeron en un cuadro resumen con el nombre del indicador, la descripción, el método, la escala y la referencia respectiva. 
Marilyn Romero-Vargas - Tania Bermúdez-Rojas - Maritza Duque-Gutiérrez

Evaluación cualitativa de indicadores de sostenibilidad socioambiental para su selección y aplicación en ciudades costarricenses

Cuadro 1. Variables utilizadas en la valoración de la factibilidad.

\begin{tabular}{|l|l|}
\hline \multicolumn{1}{|c|}{ Variable de análisis } & \multicolumn{1}{c|}{ Descripción } \\
\hline Disponibilidad de datos (DD) & $\begin{array}{l}\text { Los datos necesarios para desarrollar el indica- } \\
\text { dor se encuentran disponibles o bien la toma de } \\
\text { estos es posible. }\end{array}$ \\
\hline $\begin{array}{l}\text { Utilidad del indicador en instituciones munici- } \\
\text { pales (UIIM) }\end{array}$ & $\begin{array}{l}\text { El contenido o alcance del indicador repre- } \\
\text { senta un aspecto de interés en instituciones } \\
\text { municipales. }\end{array}$ \\
\hline $\begin{array}{l}\text { Factibilidad para la toma de datos en una sola } \\
\text { época (TDSE) }\end{array}$ & $\begin{array}{l}\text { La toma de datos necesarios para generar el } \\
\text { indicador se puede realizar en una sola estación } \\
\text { (es independiente de la época seca o lluviosa) }\end{array}$ \\
\hline $\begin{array}{l}\text { Aplicabilidad en gestión ambiental y planifica- } \\
\text { ción urbana (AGAPU) }\end{array}$ & $\begin{array}{l}\text { El indicador puede aplicarse en gestión ambien- } \\
\text { tal y urbana. }\end{array}$ \\
\hline $\begin{array}{l}\text { Incidencia positiva en la calidad ambiental } \\
\text { (IPCA) }\end{array}$ & $\begin{array}{l}\text { El indicador representa una alternativa que } \\
\text { puede repercutir positivamente en la calidad } \\
\text { del ambiente, o sea tiene doble función no solo } \\
\text { mide la calidad, sino también su aplicación } \\
\text { puede servir para mejorar el ecosistema urbano. }\end{array}$ \\
\hline Oportunidad de la información (OI) & $\begin{array}{l}\text { El indicador representa la posibilidad de obte- } \\
\text { ner y generar información que contribuya con } \\
\text { la gestión ambiental. }\end{array}$ \\
\hline Relevancia (Re) & $\begin{array}{l}\text { El indicador es de importancia en la gestión } \\
\text { ambiental. }\end{array}$ \\
\hline Robustez (Ro) & $\begin{array}{l}\text { A través del indicador se pueden generar datos } \\
\text { e información de validez. }\end{array}$ \\
\hline Continuidad (Co) & $\begin{array}{l}\text { Se cuenta con la información para que el indi- } \\
\text { cador pueda ser mantenido a través del tiempo } \\
\text { o bien la característica a medir lo permite. }\end{array}$ \\
\hline
\end{tabular}

Fuente: Morales-Cerdas, V. 2017 construido a partir de: (Rueda, 1999, Niemi y McDonald, 2004, Feria, 2003 y Hernández, 2009)

\section{Resultados y discusión}

Para orientar las ciudades hacia la sostenibilidad y la resiliencia ecológica se requiere, como señala Bai (2016), integrar la metáfora de la ciudad como un organismo (Ecosistema Urbano), con el concepto de ciudad como un sistema sociológico complejo humano-dominante (Metabolismo Urbano; MU). Además, pasar del MU conceptuado como metabolismo lineal que actualmente tienen las ciudades, caracterizado por alto consumo y producción de residuos, a un MU con enfoque cíclico, propio de los sistemas complejos que en términos prácticos significa reducir la ingesta de recursos y los residuos a niveles manejables por la naturaleza, a través 
de la innovación social y tecnológica para una eficiencia metabólica, procurando de esta forma la salud humana, tal y como plantea el Panel Internacional de la ONU (IRP 2018) y que se encuentra en el objetivo 11 de los Objetivos de desarrollo Sostenible (ODS).

Los expertos identificaron 327 indicadores de sostenibilidad socioambiental, los cuales se analizaron según sus características, similitudes y objetivos, agrupándolos en 82 indicadores, distribuidos en 9 criterios (cuadro 2): agua, energía, fauna, áreas verdes urbanas (AVU), suelo, gestión ambiental, manejo de residuos sólidos y líquidos (MRS\&L), ordenamiento del territorio y sociedad \& gobernanza.

Respecto a la cantidad de indicadores, según criterio, hay cuatro de ellos que sobresalen: sociedad \& gobernanza, áreas verdes urbanas (AVU), agua y gestión ambiental. Adicionalmente, basados en el marco conceptual expuesto en este artículo, ninguno de los especialistas mencionó indicadores asociados a los criterios aire, transporte o alimentación, lo que supone falta de experiencia de los entrevistados en estos temas o poca relación conceptual con la categoría socioambiental por parte de ellos. Según Cabero \& Infante (2014) una de las desventajas que presenta el método de criterio de experto es la dificultad de establecer el grado de experiencia de los expertos, además, de la influencia que pueden tener sobre acontecimientos imprevistos, lo que puede provocar sesgo en las respuestas.

Para indagar la instrumentalización de los indicadores a través de la bibliografía no se incluyó el criterio sociedad \& gobernanza, ya que la mayoría de estos indicadores no se relacionan directamente con el eje socioambiental, por lo que el análisis de estos se realizará desde otro enfoque. De esta forma solo se indagaron 63 indicadores para su instrumentalización, de estos solo 43 contaron con una descripción detallada, con metodología, operacionalización y escala de análisis (Cuadro 2), evidenciando que no todos los expertos conocen la instrumentalización de los indicadores señalados por ellos, y su factibilidad de aplicación. Esto podría deberse al poco conocimiento y utilización de indicadores como parte del seguimiento y evaluación constante en el manejo y gestión en ciudades o la carencia de un conjunto de indicadores que permitan la planificación y contextualización de la realidad de cada ciudad, enfocada desde la perspectiva del bienestar humano y la sostenibilidad. 
Para establecer la factibilidad teórica de cada indicador para ser aplicado en ciudades costarricenses se les facilitó a los expertos una ficha técnica con la instrumentalización de 32 indicadores, debido a que el criterio de AVU que cuenta con 11 indicadores fue analizado, tanto a nivel teórico como de campo por medio de un Trabajo Final de Graduación (Morales-Cerdas, 2017) y publicado posteriormente (Morales-Cerdas et al, 2018).

La factibilidad teórica fue de tipo cualitativo, determinando, según los expertos consultados, que solo 19 indicadores son factibles para ser aplicados en ciudades costarricenses (Cuadro 2). El resto de los indicadores (24), obtuvo una evaluación menor al $80 \%$, debido a que las variables disponibilidad de datos, factibilidad para la toma de datos en una sola época, oportunidad de la información y robustez, presentaron valores bajos.

Cuadro 2. Número de indicadores agrupados por criterio, según: encuesta a expertos, instrumentalizados y con factibilidad de ser aplicados.

\begin{tabular}{|c|c|c|c|c|c|}
\hline \multirow[b]{2}{*}{ ENFOQUE } & \multirow[b]{2}{*}{ DIMENSIÓN } & \multirow[b]{2}{*}{ CRITERIO } & \multicolumn{3}{|c|}{ NÚMERO DE INDICADORES } \\
\hline & & & $\begin{array}{c}\text { Encuesta } \\
\text { a expertos }\end{array}$ & Instrumentalizado & Factibles \\
\hline \multirow{10}{*}{$\begin{array}{l}\text { SOSTENIBILIDAD } \\
\text { URBANA }\end{array}$} & \multirow[t]{10}{*}{ SOCIOAMBIENTAL } & Agua & 12 & 7 & 4 \\
\hline & & Energía & 2 & 2 & 1 \\
\hline & & Fauna & 2 & 2 & 1 \\
\hline & & $\begin{array}{l}\text { Áreas Verdes } \\
\text { Urbanas }\end{array}$ & 17 & 11 & - \\
\hline & & Suelo & 6 & 5 & 5 \\
\hline & & $\begin{array}{l}\text { Gestión } \\
\text { ambiental }\end{array}$ & 10 & 9 & 3 \\
\hline & & $\begin{array}{l}\text { Residuos sólidos } \\
\text { y líquidos }\end{array}$ & 6 & 3 & 3 \\
\hline & & $\begin{array}{l}\text { Ordenamiento } \\
\text { del territorio }\end{array}$ & 8 & 4 & 2 \\
\hline & & $\begin{array}{l}\text { Sociedad y } \\
\text { Gobernanza }\end{array}$ & 19 & - & - \\
\hline & & TOTAL & 82 & 43 & 19 \\
\hline
\end{tabular}

Fuente: Elaboración propia

Sin embargo, dentro de las observaciones más relevantes que señalaron los expertos, y que no estaban contenidas en el instrumento de evaluación, sino que surgieron en la discusión, resaltan el tema del (1) costo, (2) la escala espacial y (3) la calidad del dato. Estos 3 aspectos no 
fueron evaluados por los expertos debido a que son variables que están pensadas para ser analizadas en campo, como fue el caso del criterio AVU (Morales-Cerdas, 2017).

Los especialistas señalaron que a pesar de que los indicadores seleccionados son de gran relevancia para evaluar la sostenibilidad socioambiental de las ciudades, estos tres aspectos son fundamentales para la factibilidad de los indicadores. Para el caso de costo, aunque este es un criterio clave de selección, existe muy poca referencia bibliográfica al respecto. En todo caso, tal y como señala Escobar (2006), el indicador debe ser costo-eficiente, es decir, que debe existir una relación positiva entre el volumen de información requerido y el costo de recolección.

Otro aspecto para considerar más allá del costo del dato es la adquisición de datos, los cuales en ciertas ocasiones no son accesibles y hasta imposibles de obtener, lo que ocasiona una disminución de la calidad de los datos para los índices planteados. Como expone Tanguay; Rajaonson; Lefebvre \& Lanoie (2010), las limitaciones causadas por la accesibilidad de los datos impiden la cuantificación y la calificación específica de indicadores.

Por otro lado, la importancia de tomar en cuenta el tamaño de la ciudad y donde se pretende implementar es fundamental, ya que la selección de los indicadores considera características, tales como densidad de población, temperatura, patrones de paisaje, entre otros (Hiremath; Balachandra; Kumarc; Bansode \&.Murali, 2013). Diferentes personas que viven en diferentes lugares tienen diferentes valores. Por lo tanto, los indicadores deben ser capaces de tener en cuenta la diversidad de lugares, personas, culturas e instituciones (Food and Agriculture Organization of the United Nations, 2002).

Conceptualizar la ciudad como un socioecosistema de humanos y naturaleza, conformado por una unidad biofísica, interaccionando con diferentes actores sociales y sus instituciones que dinamizan desde adentro el sistema (Montes y Duque, 2015), en términos operativos, se requiere la delimitación espacial de la ciudad como unidad geográfica de análisis. Toda ciudad es un espacio urbano, pero no todo espacio urbano es una ciudad.

En el caso de Costa Rica, la región GAM puede ser un socioecosistema urbano, por tanto, podría definirse como la ciudad "la Gran Ciudad Costarricense", el sitio más poblado del país y estudiar su sostenibilidad socioambiental. Sin embargo, la división territorial administrativa del país (Decreto N No 40184-MGP 2017), reconoce como ciudad únicamente al 
distrito central de cada cantón -un socioecosistema urbano con una unidad física de menor tamaño-, pero con funciones económicas de mayor jerarquía. En este sentido, surge la dificultad de delimitar la ciudad para su análisis ¿se le define como el espacio geográfico compuesto por el núcleo y su mancha urbano circundante? o como ¿el territorio perteneciente al distrito primero de cada cantón? tal y como lo establece la división político-administrativa costarricense. Esta delimitación es importante, ya que esto condiciona la selección y la utilidad de los indicadores generados en su aplicación, sobre todo en espacios urbanos muy conurbados pero divididos política y administrativamente como es el caso de la GAM, donde cada municipio define o cuenta con información distinta, que dificulta dar seguimiento al desempeño de la gestión ambiental de todas las ciudades que conforman la región. A pesar de los intentos por estandarizar los indicadores, en la realidad existe una diversidad de criterios e indicadores en temas ambientales, desde los criterios e indicadores de Bandera Azul Ecológica, los indicadores de los ODS, hasta los utilizados por el Programa Estado de la Nación, sin mencionar los que utilizan las instituciones y empresas para medir su desempeño ambiental.

En el caso de los indicadores de seguimiento adoptados por Costa Rica respecto a los ODS (Ministerio de Planificación Nacional y Política Económica 2017), estos presentan diferentes unidades espaciales de análisis, según la disponibilidad de datos. La misma situación en cuanto a escalas o unidades espaciales de análisis, se presenta con los indicadores de desempeño utilizados por el Programa Estado de la Nación para abordar el análisis del desarrollo sostenible de la ciudad (Programa Estado de la Nación 2015; 2017). La mayoría de estos indicadores están pensados para trabajar a escala país, y en algunos casos a escala cantonal o distrital en menor grado, la dificultad radica en que a menor tamaño de las unidades espaciales y o funcionales, más difícil resulta la disponibilidad y el acceso a los datos, especialmente, datos recolectados y analizados por las instituciones públicas y los municipios.

Otro caso interesante es el sistema de indicadores de desarrollo sostenible elaborado e implementado por el Banco Interamericano de Desarrollo (2016), en busca de ciudades emergentes y sostenibles de Latinoamérica y el Caribe. Este sistema es uno de los que cumple de mejor manera los requisitos que se piden de cualquier indicador y cubren todos 
los ámbitos (ambiental, social y económico) del enfoque de la sostenibilidad, sin embargo, algunos de ellos parecen ser costosos y de difícil recolección de datos, así como, unidades mínimas cartografiables distintas, lo que pareciera dificultar la integración de los datos. Sin embargo, estos fueron aplicados con éxito a escala de cantón, a la capital del país San José, definida por el BID como ciudad emergente. Con base en los indicadores seleccionados a partir de las prioridades establecidas por el municipio de la lista de indicadores de BID (2016), se realizó un diagnóstico y un plan de acción para la ciudad capital, denominado "San José Ciudad: De la acción local a la sostenibilidad metropolitana" (BID \&MSJ, 2016).

La ventaja que tiene este municipio es su capacidad económica y recurso humano para contar con información disponible, situación que sería difícil en la mayoría de municipios del país.

Para finalizar, este artículo presenta un resumen de los 19 indicadores recomendados por los expertos (Cuadro 3) a partir del análisis de su factibilidad teórica, no obstante, es importante señalar la necesidad de contar con un sistema de indicadores socioambientales para ciudades costarricenses a escala cantonal y distrital, o inclusive de barrio, que permita el seguimiento en el tiempo y la comparación entre ciudades, tomando en cuenta sus particularidades y su operacionalización dentro de un contexto geográfico particular, de esta forma se plantea una serie de característica básicas para la construcción o adaptación de un "set" de indicadores para las ciudades de Costa Rica, entre los que tenemos:

- Indicadores homogenizados y reglamentados.

- $\quad$ Adaptados a las características de la ciudad, la cual requiere ser claramente definida.

- $\quad$ De bajo costo y fácil aplicación por parte del ente competente, sea municipalidad o institución gubernamental.

- $\quad$ Fácil adquisición de datos que permita la verificación de la calidad de los mismos.

- Objetivos claros enfocados hacia el bienestar humano y la sostenibilidad de las ciudades. 
Marilyn Romero-Vargas - Tania Bermúdez-Rojas - Maritza Duque-Gutiérrez Evaluación cualitativa de indicadores de sostenibilidad socioambiental para su selección y aplicación en ciudades costarricenses

\begin{tabular}{|c|c|c|c|c|c|}
\hline 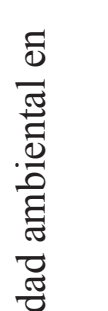 & 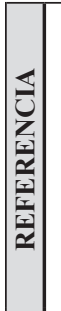 & 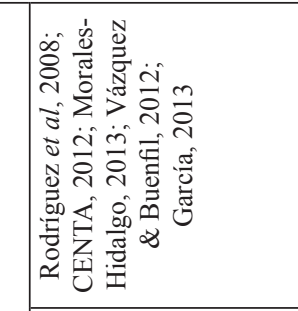 & 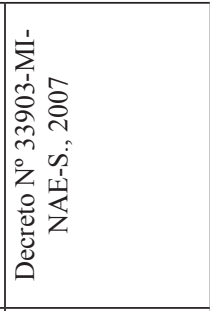 & 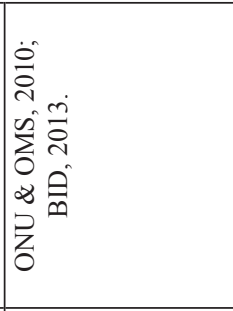 & 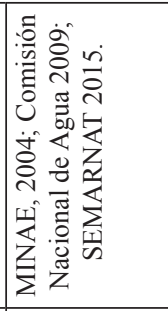 \\
\hline 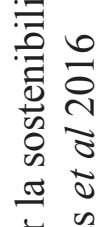 & 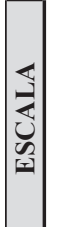 & 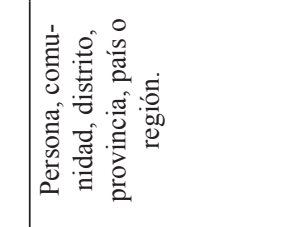 & 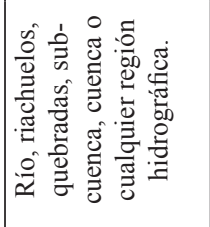 & 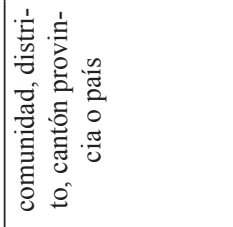 & 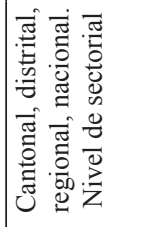 \\
\hline 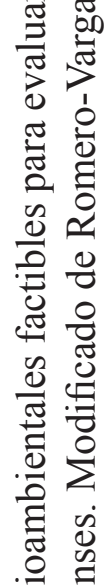 & 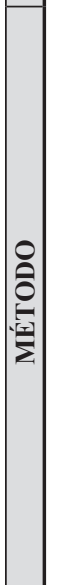 & 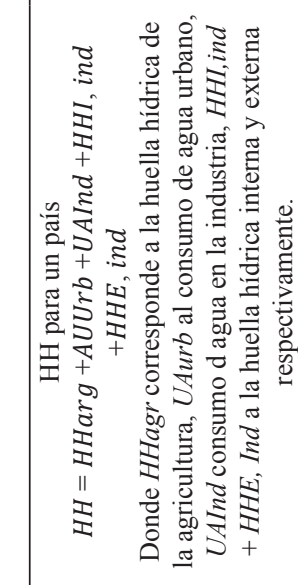 & 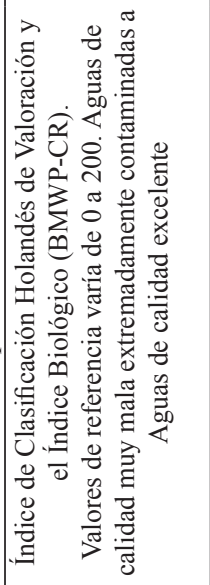 & 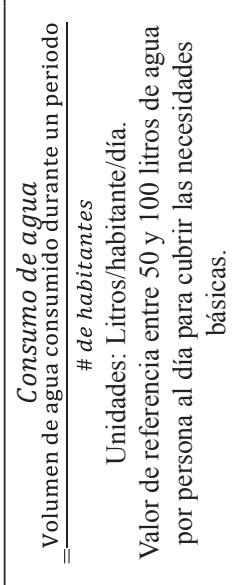 & 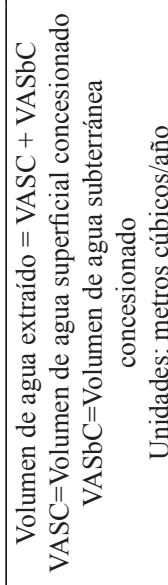 \\
\hline 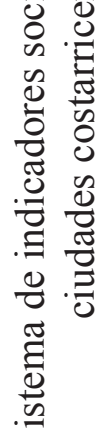 & 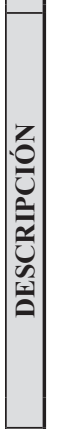 & 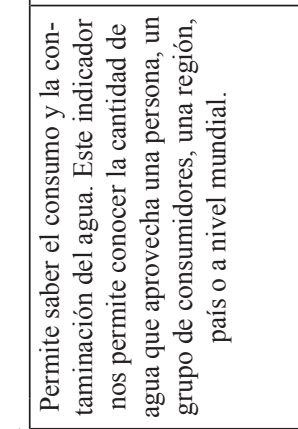 & 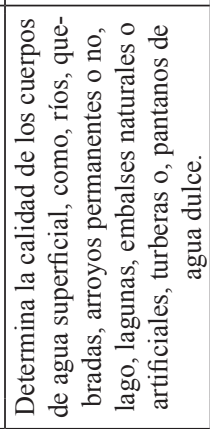 & 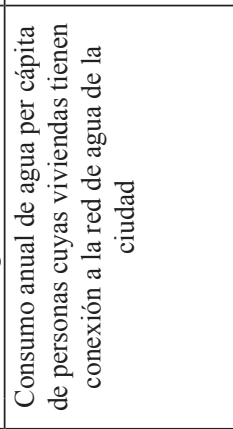 & 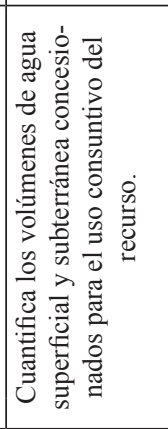 \\
\hline o & 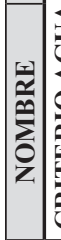 & 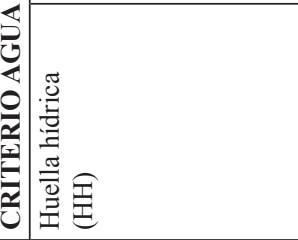 & 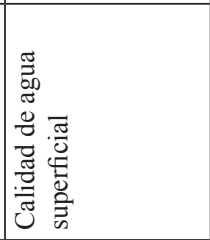 & 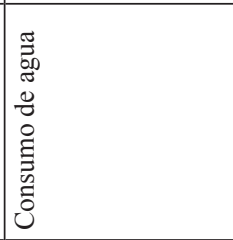 & 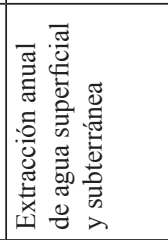 \\
\hline
\end{tabular}


Marilyn Romero-Vargas - Tania Bermúdez-Rojas - Maritza Duque-Gutiérrez

Qualitative evaluation of socio-environmental sustainability indicators $f$

or their selection and application in Costa Rican cities

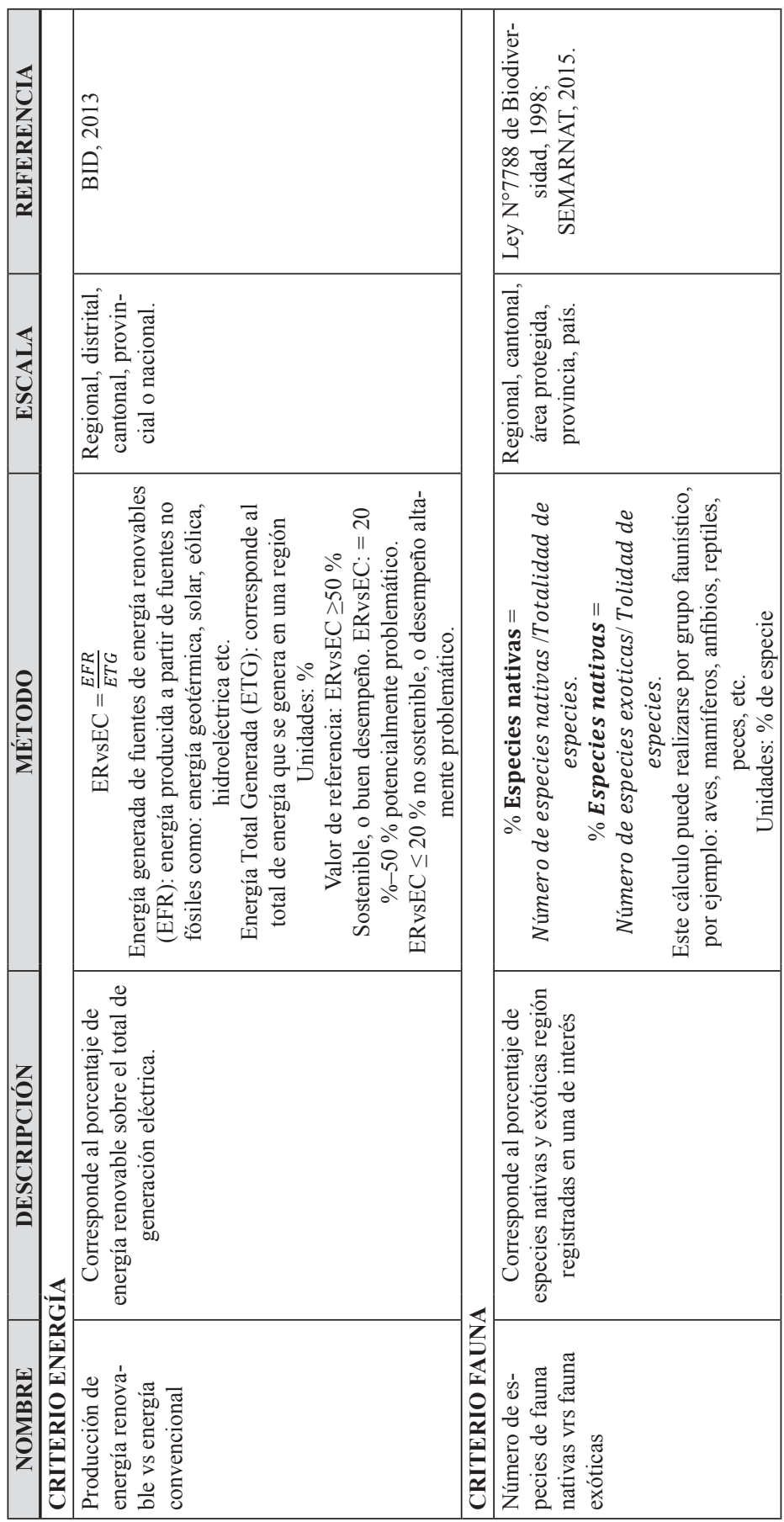


Marilyn Romero-Vargas - Tania Bermúdez-Rojas - Maritza Duque-Gutiérrez Evaluación cualitativa de indicadores de sostenibilidad socioambiental para su selección y aplicación en ciudades costarricenses

\begin{tabular}{|c|c|c|}
\hline & 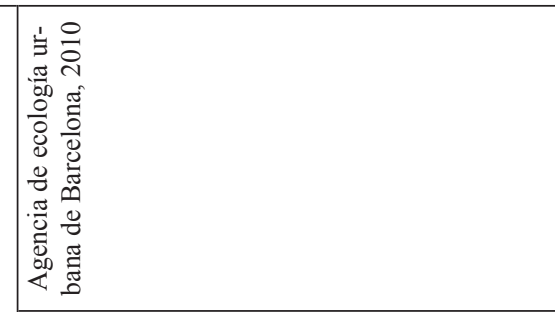 & 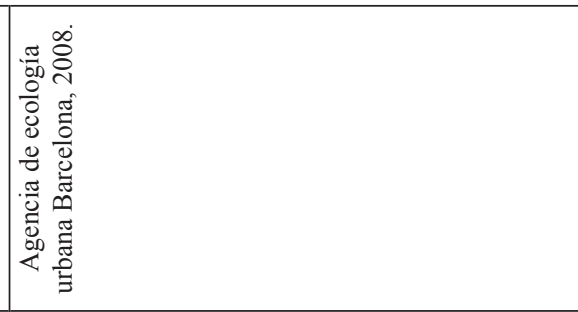 \\
\hline $\begin{array}{l}3 \\
\vdots \\
\vdots \\
0 \\
0 \\
0\end{array}$ & 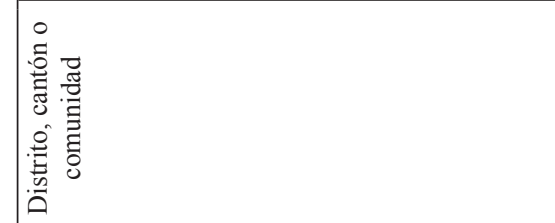 & 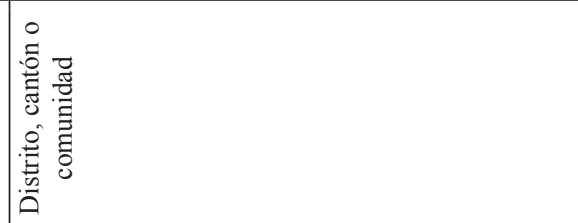 \\
\hline 을 & 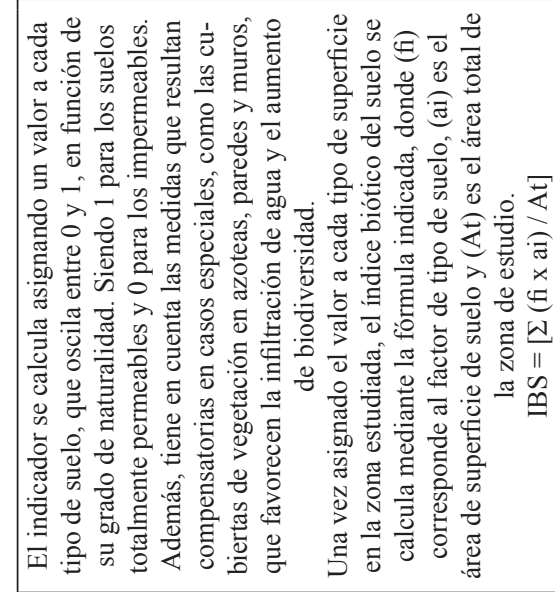 & 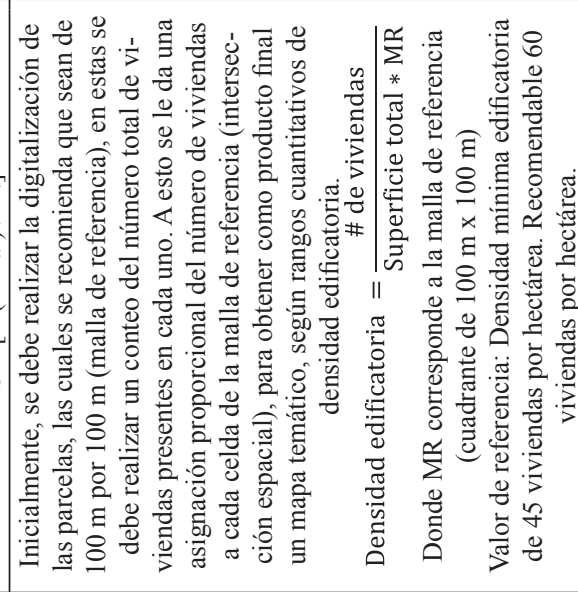 \\
\hline & 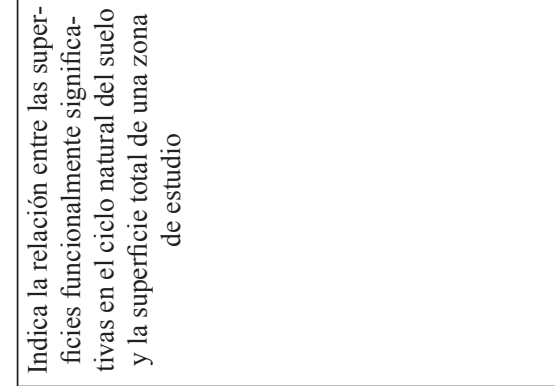 & 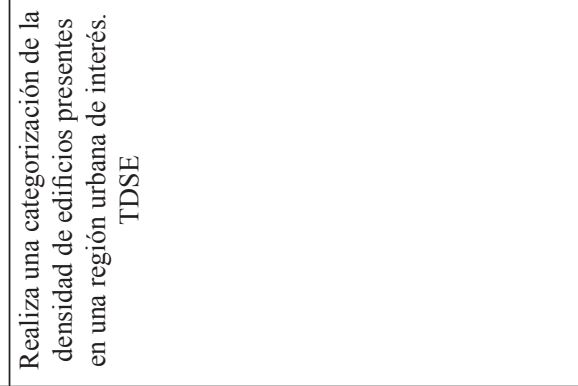 \\
\hline $\begin{array}{ll}0 \\
\\
\end{array}$ & 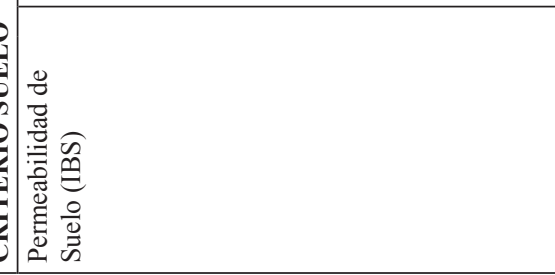 & 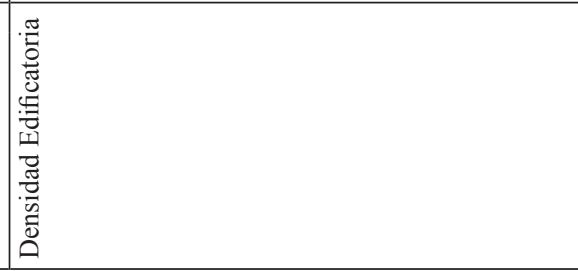 \\
\hline
\end{tabular}


Marilyn Romero-Vargas - Tania Bermúdez-Rojas - Maritza Duque-Gutiérrez

Qualitative evaluation of socio-environmental sustainability indicators $f$

or their selection and application in Costa Rican cities

\begin{tabular}{|c|c|c|c|}
\hline & 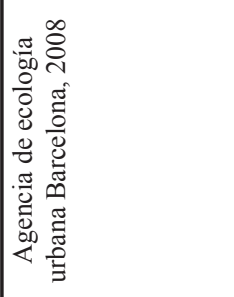 & 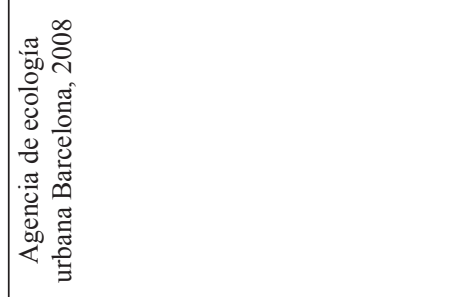 & 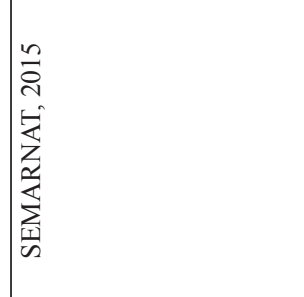 \\
\hline & 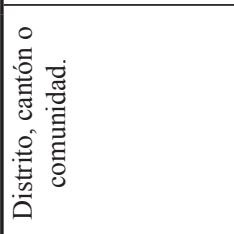 & 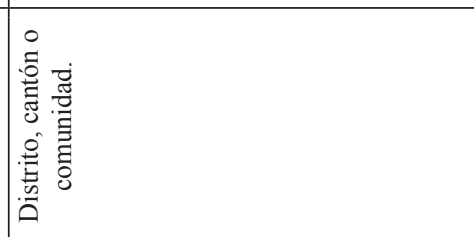 & 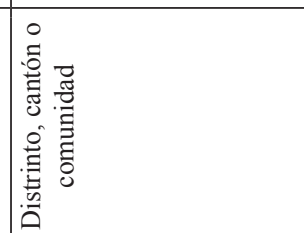 \\
\hline 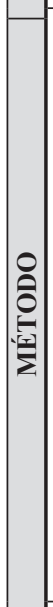 & 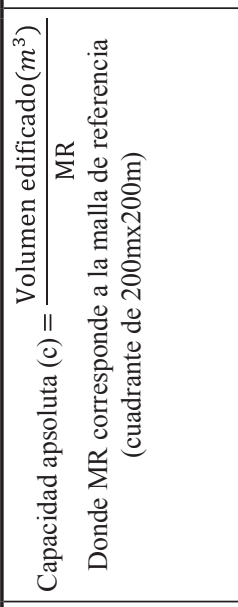 & 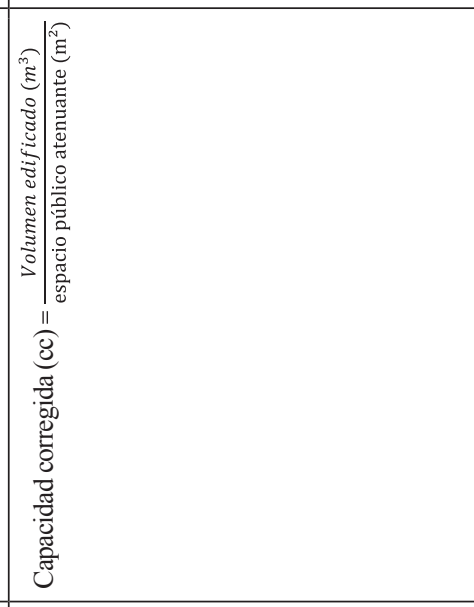 & 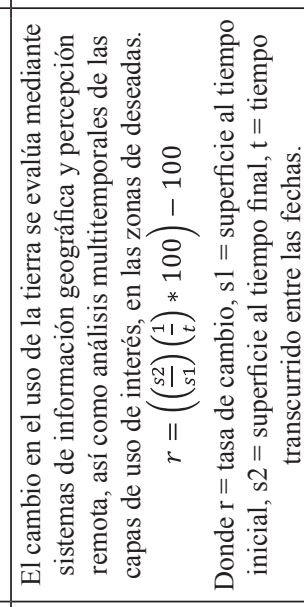 \\
\hline & 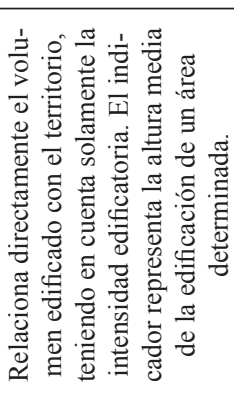 & 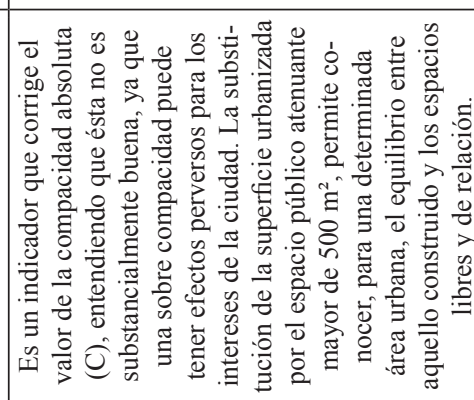 & 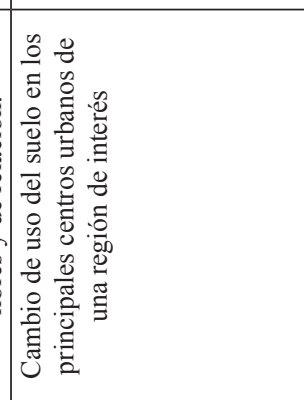 \\
\hline & 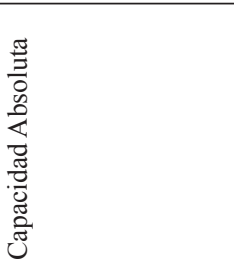 & 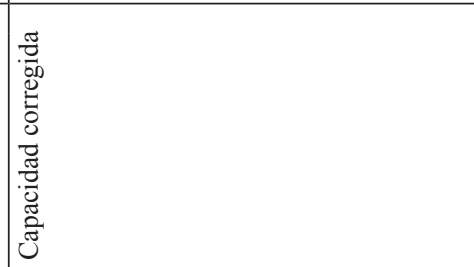 & 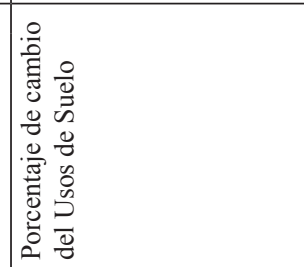 \\
\hline
\end{tabular}


Marilyn Romero-Vargas - Tania Bermúdez-Rojas - Maritza Duque-Gutiérrez Evaluación cualitativa de indicadores de sostenibilidad socioambiental para su selección y aplicación en ciudades costarricenses

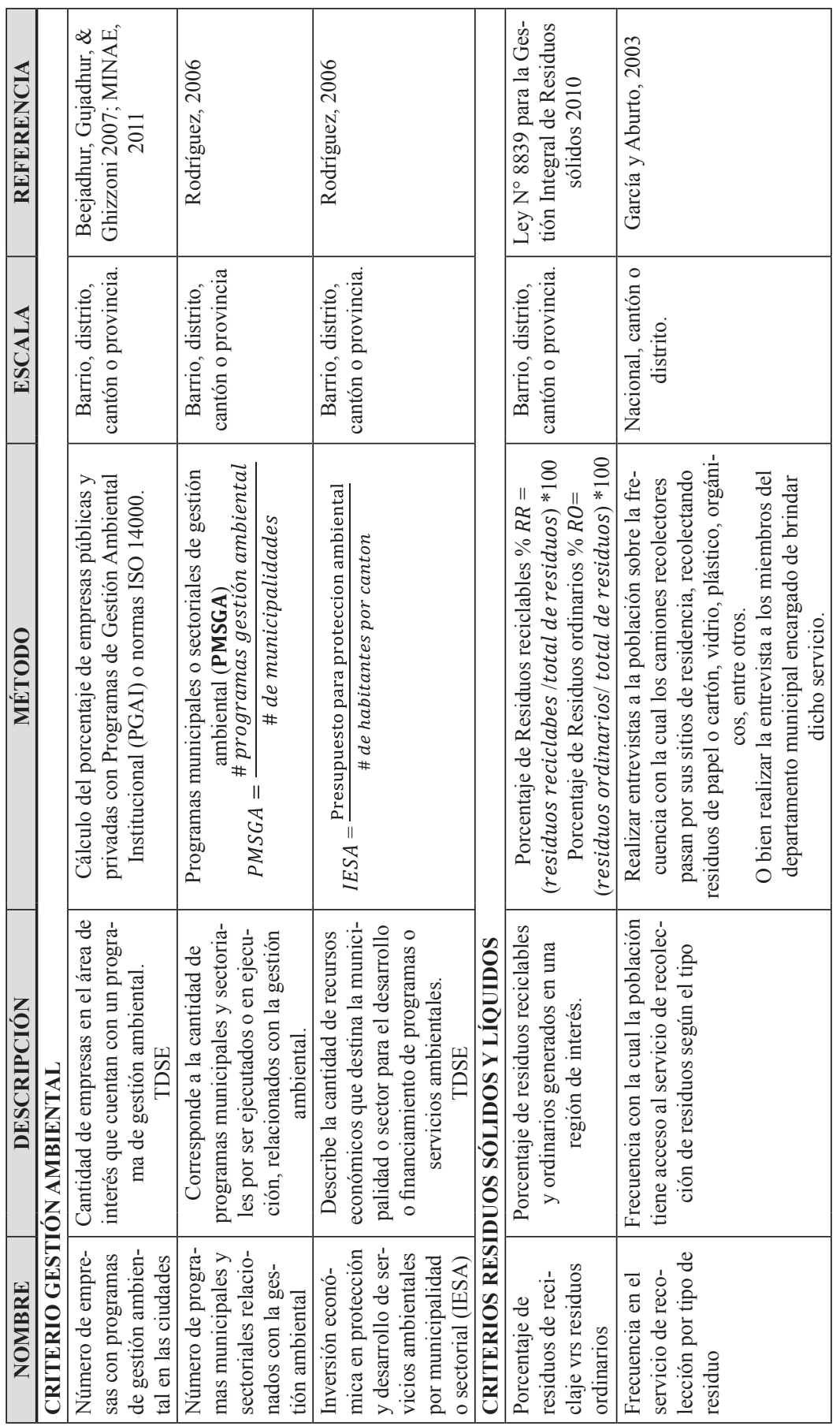


Marilyn Romero-Vargas - Tania Bermúdez-Rojas - Maritza Duque-Gutiérrez Qualitative evaluation of socio-environmental sustainability indicators $f$ or their selection and application in Costa Rican cities

\begin{tabular}{|c|c|c|c|c|}
\hline & $\begin{array}{l}n \\
\stackrel{n}{N} \\
\stackrel{n}{\infty}\end{array}$ & & 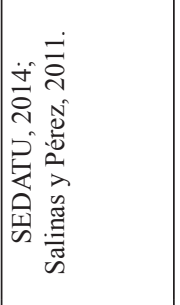 & 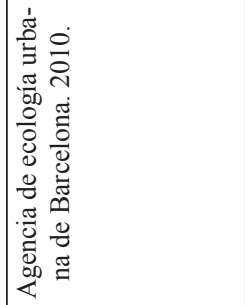 \\
\hline 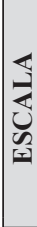 & 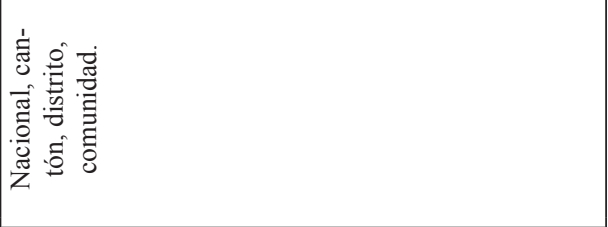 & & 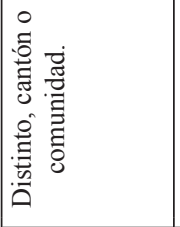 & 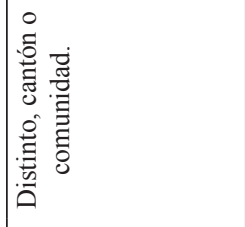 \\
\hline & 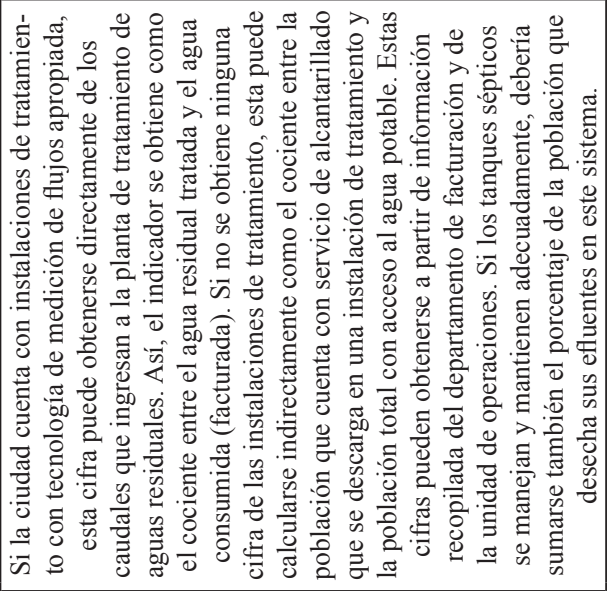 & & 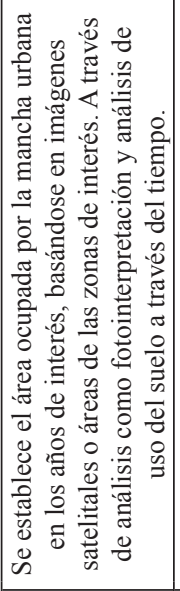 & 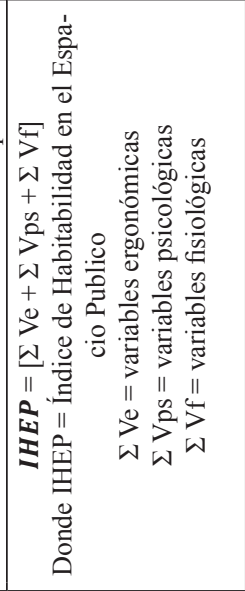 \\
\hline 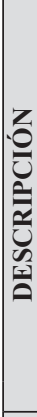 & 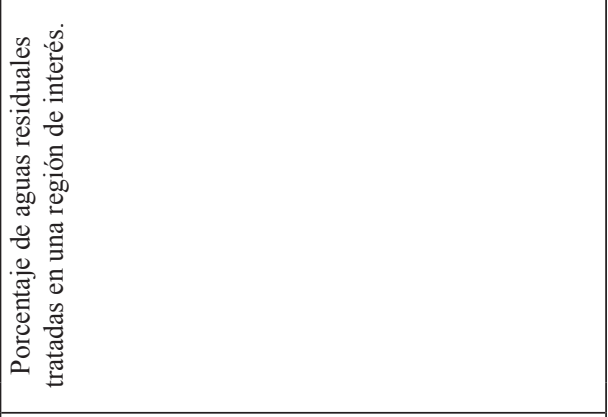 & 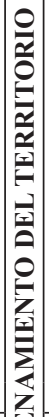 & 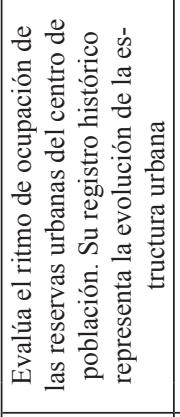 & 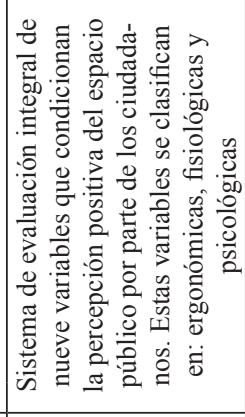 \\
\hline 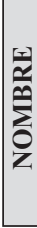 & 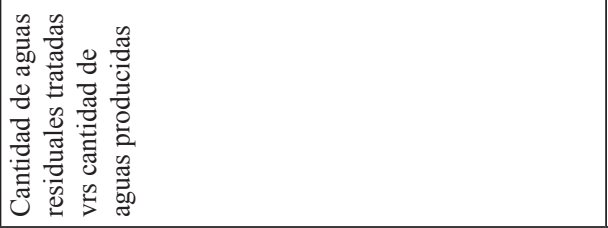 & 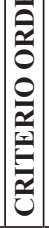 & 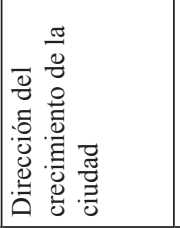 & 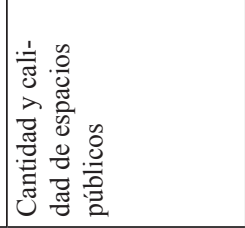 \\
\hline
\end{tabular}




\section{Conclusiones}

En este estudio el uso del criterio de experto permitió identificar, seleccionar y medir la factibilidad de indicadores socioambientales, para evaluar la sostenibilidad de las ciudades. A pesar de esta fortaleza, al realizar una búsqueda detallada de cada indicador, no todos contaban con una descripción, metodología, operacionalización y escala de análisis. Esto supone, que en caso de los expertos puede haber una falta de conocimiento en temas emergentes a nivel de país o sociedad; desconocimiento de la instrumentalización de muchos de los indicadores y poca relación conceptual con la sostenibilidad socioambiental de ciudades. Aun así, el uso de esta metodología es importante, ya que los expertos son los trabajan en el manejo y la gestión de la ciudad. Es recomendable robustecer esta metodología con comprobaciones de campo que incluya variables, tales como costo, escala espacial y calidad del dato, permitiendo así la triangulación de varios métodos.

Al evaluar la calidad del sistema urbano por medio de los indicadores, un elemento clave a tomar en cuenta es la definición conceptual y espacial de la ciudad costarricense. Aun cuando existe a nivel administrativo la definición de ciudad (se reduce solo al distrito central de cada cantón), está es muy limitada para efectos del seguimiento y la comparación, ya que excluye otros espacios y elementos que forman parte del entramado urbano. Además, como no existe una delimitación integral de la ciudad, cada planificador o gestor utiliza diferentes conceptos y escalas de análisis.

Finalmente, es recomendable concebir la ciudad como un sistema que entrelace el metabolismo urbano cíclico con el ecosistema urbano, esto permite que no solo se analicen las entradas y salidas del sistema, sino también la estructura, la función, los patrones y los procesos en/de la ciudad.

\section{Referencias}

Agencia de Ecología Urbana de Barcelona. (2008). Plan Especial de Indicadores de Sostenibilidad Ambiental de la Actividad Urbanística de Sevilla.

Agencia de Ecología Urbana de Barcelona. (2010). Plan de Indicadores de Sostenibilidad Urbana de Vitoria-Gasteiz.

Asamblea Legislativa de la República de Costa Rica (1998). Ley de Biodiversidad. [Ley No7788]. La Gaceta No 101 del: 27/05/1998. 
Asamblea Legislativa de la República de Costa Rica (2010). Ley para la Gestión Integral de Residuos sólidos. [Ley $\mathrm{N}^{\circ}$ 8839]. La Gaceta $\mathrm{N}^{\circ}$ 135 del 13/07/2010.

Bai, X. (2016). Eight energy and material flow characteristics of urban ecosystems. Ambio 45,819-830 Doi 10.1007/s13280-016-0785-6

Banco Interamericano de Desarrollo (BID). (2016). Indicadores de la Iniciativa Ciudades Emergentes y Sostenibles, Guía metodológica. Anexo (Pdf). Disponible en https://publications.iadb.org/publications/spanish/document/[Recuperado 4/01/2019]

Banco Interamericano de Desarrollo (BID) y Municipalidad de San José. (2016). San José, de la acción local a la sostenibilidad metropolitana (Pdf). San José, Costa Rica. Disponible en https://www.msj. go.cr/MSJ/Capital/SiteAssets/ [Recuperado 4/01/2019]

Beejadhur, Y., Gujadhur, S. \& Ghizzoni, L. (2007). Introducción a ISO 14000, sistemas de gestión ambiental. Boletín 78.

Blasco, J.; López, A.; Mengual, S. (2010). Validación mediante método delphi de un cuestionario para conocer las experiencias e interés hacia las actividades acuáticas con especial atención al windsurf. Ágora para la educación y el deporte 12(1),75-96.

Cabero, J. \& Infante, A. (2014). Empleo del método Delphi y su empleo en la investigación en comunicación y educación. EDUTEC. Revista Electrónica de Tecnología Educativa, №48. Recuperado de http:// edutec.rediris.es/Revelec2/Revelec48/n48_Cabero_Infante.html

CENTA. (2012). Día mundial del agua: material didáctico sobre la huella hídrica, la huella del agua.

Comisión Nacional del Agua (CONAGUA). (2009). Estadísticas del Agua de la Región Hidrológico-Administrativa XIII, Aguas del Valle de México. México.

Consejo Nacional de Planificación Urbana. (2013). Plan GAM 2013-2030. Informe técnico. Recuperado de http://www.mivah.go.cr.

Corral, Y., Corral, I., y A. Franco. (2015). Procedimientos de muestreo. Revista Ciencias de la Educación 26 (46), 151-167. Recuperado de http://servicio.bc.uc.edu.ve/educacion/revista/46/art13.pdf. [Recuperado 16/05/2019]

Crutzen, P. J. (2010). Anthropocene Man. Nature International Journal of Science (pdf), 467(7317): S10. http://doi.org/10.1038/4675S10a. 
Marilyn Romero-Vargas - Tania Bermúdez-Rojas - Maritza Duque-Gutiérrez Evaluación cualitativa de indicadores de sostenibilidad socioambiental para su selección y aplicación en ciudades costarricenses

Escobar, L. (2006). Indicadores sintéticos de calidad ambiental: un modelo general para grandes zonas urbanas. EURE 32 (96): 73-98.

FAO. (2002). Pressure-State-Response Framework and Environmental Indicators. Recuperado de http://www.fao.org/ag/againfo/programmes/en/lead/toolbox/refer/envindi.htm [Accessed July 2018].

Feria, T. (2003). Indicadores de sustentabilidad: un instrumento para la gestión urbana. López Trigal, L. et al., coord. La ciudad. Nuevos procesos, nuevas respuestas. Universidad de León-AGE, 241-253.

García, A. (2013). La huella hídrica como indicador de presiones: aplicación a la cuenca del Duero y al sector porcino español. Universidad de Alcalá. Madrid, España

García, L. y Aburto, A. (2003). Recolección y Tratamiento de Desechos Sólidos Manuales Elementales de Servicios Municipales. El Instituto Nicaragüense de Fomento Municipal (INIFOM). Nicaragua.

Gleeson, B. (2013). What role for social science in the 'urban age'? International Journal of Urban and Regional Research 37 (5), 1839-51.

Hernández, A. (2009). Calidad de vida y medio ambiente urbano. Indicadores locales de sostenibilidad y calidad de vida urbana. Revista INVI, 65 (24), 79-111.

Hiremath, R. B; Balachandra, P.; Kumar, B.; Bansode, S. S. \& Murali, J. (2013). Indicator based urban sustainability. A review. Energy for Sustainable Development 17, 555- 563. https://doi.org/10.1016/j. esd.2013.08.004

Instituto Nacional de Estadísticas y Censos. (2018). Costa Rica en cifras (Pdf). Disponible en www.inec.go.cr/sites/documetos-bibliotecavirtual/, [Recuperado 25/01/19

IRP (2018). The Weight of Cities: Resource Requirements of Future Urbanization. Swilling, M., Hajer, M., Baynes, T., Bergesen, J., Labbé, F., Musango, J.K., Ramaswami, A., Robinson, B., Salat, S., Suh, S., Currie, P., Fang, A., Hanson, A., Kruit, K., Reiner, M., Smit, S., Tabory, S. A. Report by the International Resource Panel. United Nations Environment Programme, Nairobi, Kenya.

Luna, P.; Infante, A.; Martínez, F. J. (2005). Los Delphi como fundamento metodológico predictivo para la investigación en sistemas de información y tecnologías de la información (IS/IT). Revista de Medios y Educación 26,89-112. 
Ministerio de Planificación Nacional y Política Económica, Secretaría Técnica de los ODS. (2017). Objetivos de desarrollo Sostenible: Indicadores de seguimiento, Costa Rica (PDF). MIDEPLAN; INEC; PNUD-San José, CR. Disponible en www.mideplan.go.cr Recuperado [24-01-2019]

MINAE. (2011). Guía para la elaboración de programas de gestión ambiental Institucional (PGAI) en el sector público de Costa Rica: documento de orientación para las instituciones públicas. MINAET, Ministerio de Salud. San José, Costa Rica: CYMA, USAID, CCAD, GIZ.

MINAE. (2004). Manual técnico del departamento de aguas. La Gaceta No 98 del 20/05/2004

MINAE-S. (2007). Reglamento para la Evaluación y Clasificación de la Calidad de Cuerpos de Agua Superficiales. [Decreto No 33903]La Gaceta diario oficial, Costa Rica, 17 de setiembre del 2007.

MGP. (2017). (2017). División Territorial Administrativa de la República de Costa Rica. Editorial Imprenta Nacional. [Decreto No 40184]La Gaceta diario oficial N 48. Disponible en https://www.imprentanacional.go.cr/editorialdigital/libros/historiaygeografia/division_17. [Recuperado 24/01/2019].

Montes, C. y Duque, M. (2015). Ciudades resilientes en el Antropoceno; mito o realidad. Ciudad y Territorio. Estudios Territoriales. CyTET XLVII (183).

Morales-Cerdas, V., Piedra Castro, L., Romero Vargas, M. \& Bermúdez Rojas, T. (2018). Indicadores ambientales de áreas verdes urbanas para la gestión en dos ciudades de Costa Rica. Revista de Biología Tropical, 66(4), 1421-1435.

Morales-Cerdas, V. (2017). Indicadores de calidad ambiental a partir del criterio áreas verdes urbanas: aplicación en dos ciudades de Costa Rica. (Tesis de Licenciatura). Universidad Nacional, Heredia, Costa Rica.

Morales-Hidalgo, D. (2013). Costa Rica. En J. Mahlknecht. \& E, PasténZapata. Diagnóstico de los recursos hídricos en América Latina (221-245p). México: Pearson Education.

Munier, N. (2011). Methodology to select a set of urban sustainability indicators to measure the state of the city, and performance assessment. Ecological Indicators, 11(5), 1020-1026. 
Marilyn Romero-Vargas - Tania Bermúdez-Rojas - Maritza Duque-Gutiérrez Evaluación cualitativa de indicadores de sostenibilidad socioambiental para su selección y aplicación en ciudades costarricenses

Niemi, G. \& McDonald, M. (2004). Application of Ecological Indicators. Annu. Rev. Ecol. Evol. Syst. 35, 89-111.

Odum, E. P. (1971). Fundamentals of Ecology. Third Edition, W.B. Saunders Co., Philadelphia.

Organización de las Naciones Unidas (ONU) y Organización Mundial de la Salud (OMS). (2010). El derecho al agua. folleto informativo 35. PLAN-MINAE-MIVAH-MOPT-S-MAG (2014). Oficializa el Plan Regional de Ordenamiento Territorial de la Gran Área Metropolitana, Plan GAM 2013-2030. [Decreto No 38145]. Gaceta N82. Recuperado de http://www.pgrweb.go.cr [recuperado 01/02/2019

Programa Estado de la Nación (2015). Informe Estado de la Nación 2015. San José Costa Rica. Recuperado de www.estadonacion.or.cr

Programa Estado de la Nación (2017). Informe Estado de la Nación 2017.

San José, Costa Rica. Recuperado de www.estadonacion.or.cr

Quiroga, R. (2009). Guía metodológica para desarrollar indicadores ambientales y de desarrollo sostenible en países de América Latina y el Caribe. CEPAL. Manual No 61.

Rodríguez, R., Garrido, A., Llamas, R. \& Varela, C. (2008). La huella hidrológica de la agricultura española. Papeles de Agua Virtual, 2. 22p. Rodríguez, G. (2006). Décimo tercer informe Estado de la Nación en desarrollo humano sostenible: Gestión Ambiental Municipal y Participación Local. Estado de la Nación, Costa Rica.

Romero-Vargas, M., Bermúdez-Rojas, T., Piedra-Castro, L. \& MoralesCerdas, V. (2016). Criterios e Indicadores de Sostenibilidad Socioambiental para Ciudades de Costa Rica. Informe Técnico. Universidad Nacional, Heredia, CR.

Rueda, S. (1999). Modelos e indicadores para ciudades más sostenibles. Taller sobre Indicadores de Huella y Ambiental Urbana. Barcelona. España.

Rueda, S., De Cáceres, R., Cuchí, A., \& Brau, L. (2012). El urbanismo ecológico:su aplicación en el diseño de un ecobarrio en Figueres. Barcelona: BCN ecología (Agencia de Ecología Urbana).

Salinas, E. y Pérez, L. (2011). Procesos urbanos recientes en el Área Metropolitana de Concepción: transformaciones morfológicas y tipologías de ocupación. Rev. geogr. Norte Gd. [online]. 49, 79-97. 
Secretaría de Medio Ambiente y Recursos Naturales (SEMARNAT). (2015). Indicadores básicos del desempeño ambiental en México. Suelo. http://app1.semarnat.gob.mx/dgeia/indicadores14/conjuntob/03_suelos/03_suelos_esquema.html. [Recuperado julio 2017].

Secretaria de Desarrollo Agrario, Territorial y Urbano, México (SEDATU). (2014). Sistema de Indicadores de Evaluación y Seguimiento Programa de Desarrollo Urbano del Centro de Población de Cancún 2014-2030.

Tanguay, G. A., Rajaonson, J., Lefebvre, J.F. \& Lanoie, P. (2010) Measuring the sustainability of cities: An analysis of the use of local indicators. Ecological Indicators 10, 407-418.

Vázquez, R. \& Buenfil, M. (2012.). Huella Hídrica de América Latina: retos y oportunidades. Aqua-LAC, 4 (1), $41-48$ p. 
